\title{
Les haches à douille armoricaines revisitées
}

José Gomez de Soto

\section{OpenEdition}

\section{Journals}

Édition électronique

URL : http://journals.openedition.org/rao/2225

DOI : $10.4000 /$ rao.2225

ISBN : 978-2-7535-3432-2

ISSN : 1775-3732

Éditeur

Presses universitaires de Rennes

Édition imprimée

Date de publication : 25 décembre 2013

Pagination : 313-315

ISBN : 978-2-7535-3430-8

ISSN : 0767-709X

\section{Référence électronique}

José Gomez de Soto, «Les haches à douille armoricaines revisitées », Revue archéologique de l'Ouest [En ligne], 30 | 2013, mis en ligne le 25 décembre 2013, consulté le 04 décembre 2020. URL : http:// journals.openedition.org/rao/2225 ; DOI : https://doi.org/10.4000/rao.2225

Ce document a été généré automatiquement le 4 décembre 2020.

Tous droits réservés 


\title{
Les haches à douille armoricaines revisitées
}

\author{
José Gomez de Soto
}

\section{RÉFÉRENCE}

Rivallain J., 2012 - Les haches à douille armoricaines revisitées. Apports des travaux et des études de la deuxième moitié du $x x^{e}$ siècle en Bretagne, Les Dossiers du Centre régional d'Archéologie d'Alet, suppl. A1, 2012, 224 p., 109 fig., 1 cédérom. (ISSN 0899-662)

\footnotetext{
"Les haches à douille de type armoricain sont un élément majeur du premier âge du Fer de Bretagne et de Normandie [....] elles sont rarement associées à d'autres objets sinon à des éléments du premier âge du Fer. Les associations anciennement signalées dans les dépôts en langue de carpe ne sont pas confirmées de nos jours, c'est pourquoi les haches à douille de type armoricain doivent être détachées de l'âge du Bronze et considérées comme une entité originale du début de l'âge du Fer. "

J. Briard, in Documents d'Archéologie méridionale, 24, 2001, p. 261.
}

1 Notre regretté collègue et ami Jacques Briard avait fait projet de reprendre de fond en comble - et ce à l'échelle européenne - la question des haches à douille de type armoricain, et pour ce faire avait commencé à rassembler une équipe ; mais sa maladie et sa trop rapide disparition empêchèrent que ce beau projet pût être mené à bonne fin. La problématique des haches à douille de type armoricain était de ce fait restée ouverte.

2 Par le présent ouvrage, Josette Rivallain propose une synthèse sur les haches à douille de type armoricain - limitée aux seules trouvées en Bretagne - poursuivant ainsi la recherche entreprise avec sa thèse publiée en 1971.

3 Une première remarque concernera le titre: sémantiquement, une hache à douille armoricaine est une hache à douille fabriquée ou trouvée en Armorique, quel qu'en soit le type. Aussi, faudrait-il plutôt écrire « haches à douille de type armoricain ", l'adjectif «armoricain» ayant valeur d'éponyme sans préjuger du lieu de production ou de trouvaille: il est bien connu que les haches qui nous intéressent furent aussi très 
abondamment produites en Normandie, et qu'un certain nombre s'exportèrent à l'époque plus ou moins plus ou moins loin de ces deux régions, telles deux inédites découvertes depuis peu dans les Deux-Sèvres.

4 L'ouvrage se lit agréablement. On regrettera toutefois une certaine négligence à la relecture (ex., P. du Chatellier et Paul Du Chatelier p.15, devenant P. du Châtelier p. 122 ; p. 74 « petites haches de type Brandivy, en miniature » alors que les haches du type de Brandivy sont les plus grandes et massives de toutes, ce qu'évidemment l'auteure n'ignore pas!), des coquilles trop nombreuses, des appels incomplets pour certaines figures (ex., p. 199) ou appels bibliographiques (ex., p. 147 : « Carfantain [Dolde-Bretagne, 35, 1887] »). Quelques légendes de figures sont inexactes ou incomplètes : fig. 80, les dessins attribués à Louis Marsille, qui sont d'Yvan Onnée; les fig. 75 et 76 mélangeant objets associés à des dépôts de haches à d'autres n'en venant pas (Dol-deBretagne, le Rocher au Bono, Plougoumelen), sans que le fait soit précisé pour ces derniers.

5 La bibliographie (qui comporte un nombre important de travaux non appelés dans le texte...), a été partagée selon trois thèmes différents, ce qui n'en facilite guère la consultation. On regrettera aussi l'absence d'index.

6 L'iconographie, riche et de bonne qualité générale, fournit un nombre très important de dessins de haches inédits, tant des types armoricains que des modèles antérieurs du Bronze final atlantique 3 (BFa 3). Cependant, on déplorera quelques inutiles reproductions de très mauvais croquis, ex. fig. 76 (objets du dépôt de Plonéis, par ailleurs déjà figurés par d'excellents dessins sur la fig. précédente) ou fig. 85 (dépôt de Questembert).

7 Le premier chapitre de l'ouvrage (p. 5-35) est consacré à l'historique des trouvailles de haches à douille de type armoricain et de l'intérêt que leur portèrent le monde érudit, les sociétés savantes et les musées. Ainsi sont évoquées les grandes figures du président de Robien, de Paul Maufras du Chatellier, Louis Marsille, Gabriel de Mortillet, Joseph Déchelette, et de bien d'autres. Un hommage mérité est rendu à Pierre-Roland Giot. Le rôle fondamental de Jacques Briard pour l'élaboration des protocoles d'étude et d'interprétation modernes des haches à douille de type armoricain est justement souligné. Mais on reste étonné que l'évocation de Jacques Briard se limitât à sa thèse de 1965 , négligeant les premiers doutes qu'il formula quant à la date des haches à douille de type armoricain dès 1991 (actes du colloque de Beynac) puis sa drastique et sans appel remise en question chronologique de 2001 (cf. citation en tête de ce compterendu)!

Le chapitre 2 (p. 37-153) forme le corps de l'ouvrage. La typologie des haches à douille de type armoricain, présentée en premier (p. 37-50), est une reprise de celle élaborée par Jacques Briard dans son ouvrage de 1965, que complétèrent les fiches typologiques préparées par ce dernier en collaboration avec Guy Verron publiées par la Société préhistorique française en 1976. Puis les conditions de découverte des dépôts sont abordées, département par département (p. 51-81), avec des données aussi détaillées que possible: se trouvent ainsi commodément réunies de précieuses informations dispersées dans les revues locales et les archives. Ensuite est fait retour à la typologie et aux mesures des haches, avec un intérêt particulier accordé aux décors, qui font l'objet d'une solide analyse (p. 81-119). Puis les étapes de la fabrication des haches sont présentées, mais les moules figurés en illustration n'étaient cependant pour la plupart pas destinés à la production de haches de types armoricains, mais de modèles plus 
anciens du BFa 3 (p. 120-140). Enfin, la partie «Les associations » (p. 140-153) traite des associations des haches avec des objets d'autres types, mais aussi des conditions d'enfouissement et de l'environnement des dépôts. Ici (p. 146-149) sont rappelés les fragments de bracelets à bossettes associés aux dépôts de haches à douille de type armoricain (dont les fragments inédits d'un bracelet à bossettes globuleuses de LocoalMendon, typique du Hallstatt D1, fig. 77). L'auteure ne différencie pas ces bracelets de ceux des dépôts de l'horizon de l'épée en langue de carpe du BFa 3, pourtant typologiquement différents comme le montre un échantillon de ces derniers fig. 107. Quant à la date C14 de Saint-Bugan, évoquée p. 150 puis à nouveau dans la conclusion p. 202 (mais là attribuée au dépôt de Tréhou...), Gsy-42, 2518 +/- 130 BP, elle présentée par sa seule date centrale brute, sans tenir compte de sa nécessaire calibration.

9 Le chapitre 3 (p. 154-188) est consacré aux haches à douille de type non armoricain. Il s'agit ici de celles de l'horizon de l'épée en langue de carpe du BFa 3. Comme pour les dépôts de haches de type armoricain, se trouvent ici réunies des informations dispersées dans les revues et les archives. De ces haches à douille du BFa 3, il a été déjà largement traité par nombre d'auteurs, en particulier Jacques Briard (Les dépôts bretons..., Rennes, 1965 et travaux postérieurs), par ce dernier et Guy Verron (Typologie..., Paris, SPF, 1976), et tout récemment par Pierre-Yves Milcent (Le temps des élites en Gaule atlantique..., Rennes, 2012). On reste pour le moins étonné d'affirmations erronées: les dépôts interprétés comme un rassemblement hétéroclite d'objets d'époques diverses (p. 187-188, puis dans la conclusion p. 199), ou l'épée pistilliforme présentée comme le type dominant de la période (p. 165). Étonne encore l'utilisation de la notion obsolète de Cultures des Champs d'Urnes (p.187), et plus loin dans la conclusion, de celle également obsolète du Plainseau (p. 199). Et un moule pour hache à ailerons se voit présenté comme moule pour hache à douille (p. 183 et fig. 108)! L'auteure mentionne sans commentaire (p. 187) les haches à douille de type armoricain prétendument trouvées dans quelques dépôts de l'horizon de l'épée en langue de carpe, sans tenir compte de l'examen critique des sources qui a montré - constat qui fait désormais consensus - qu'il ne s'agit que de la conséquence de mélanges de collection : le cas est particulièrement flagrant pour le dépôt de l'île Verte à L'île de Batz contesté dès 1883 par Victor Micault, mais mis en exergue ici !

Le chapitre 4 (p. 189-197) intitulé «Le contexte des haches à douille» revient sur les conditions d'enfouissement des haches, et aborde des sujets divers avec une documentation concernant essentiellement... diverses périodes de l'âge du Bronze. En ce qui concerne les métaux, l'auteure rappelle avec raison (p.192) qu'en Bretagne " pour la période protohistorique, nos connaissances de l'exploitation minière est (sic) pratiquement inexistante ». C'est également dans ce quatrième chapitre qu'est traitée la question de la fonction des haches à douille (p.194-197), en particulier la notion certainement pertinente d'accumulation de richesse. On est cependant étonné d'une affirmation telle que "[les haches du type du] Plainseau, centrées sur l'Escaut et la Somme » alors que ces haches, fonctionnelles au contraire de celles de type armoricain, représentent le type de loin le plus commun de tout l'arc atlantique, du Benelux à l'Aquitaine septentrionale (par ex., plus de 20 ex. intactes et 50 fragments dans le dépôt de Vénat en Charente).

11 La conclusion (p. 199-202), après un nouvel et juste hommage rendu à Jacques Briard, insiste à nouveau sur les deux groupes distincts de haches à douille de Bretagne, celles des dépôts de l'horizon de l'épée en langue de carpe et celles de type armoricain. Les 
cartes présentées plus haut p.154 illustraient bien en effet leurs répartitions différenciées, celle des dépôts de haches à douille de type armoricain occupant plus largement l'intérieur des terres. Mais il est désormais démontré qu'il s'agit de deux phénomènes non contemporains, le premier datable du BFa 3 vers 950-800 av. J.-C., le second du premier âge du Fer, donc postérieur à env. 800 av. J.-C.! Bien qu'actuellement documentée pour le seul $\mathrm{Ha} \mathrm{D}$, c'est à dire du milieu du $\mathrm{VII}^{\mathrm{e}} \mathrm{s}$. au milieu du ve $\mathbf{e}$. av J.-C., la production de ces haches a pu débuter dès le Ha C, au viII ${ }^{\mathrm{e}} \mathrm{s}$. av. J.-C. Cet état du savoir fut bien affirmé, comme nous l'avons rappelé ci-dessus, dès 1991 puis en 2001 par Jacques Briard, et confirmé depuis par l'analyse critique des trouvailles anciennes et la récente découverte de Kergariou à Quimper. Et, preuve supplémentaire, le métal des objets des dépôts de l'horizon de l'épée en langue da carpe et celui des haches à douille de type armoricain venant de la même région montrent des compositions chimiques exclusives les unes des autres (Le Carlier de Veslud et al., SAO, 2009 et Journée du CReAAH, 2011, p. 48), mais aussi que, pour le dépôt de Trelly, les haches et les parures du Ha $\mathrm{D}$ associées présentent des compositions tout à fait compatibles indice sérieux de leur contemporanéité (Aranda et al., Bull. Soc. préh. fr, 2013, p. 117). Le phénomène des dépôts de haches à douille de type armoricain n'est d'ailleurs qu'un des aspects d'un phénomène plus général de réémergence, ou du moins de sa multiplication, de la pratique des dépôts métalliques terrestres au cours du premier âge du Fer, illustrée en Centre-Ouest, dans le Bassin parisien ou encore avec le Launacien du Languedoc. L'auteure, pour qui « la fin de l'âge du Bronze en Bretagne reste connue par la grande quantité de dépôts d'objets en bronze : les haches à douille armoricaines ont été produites à travers toute la Bretagne... » (p. 189), en persistant à présenter dépôts de l'horizon de l'épée en langue de carpe et dépôts de haches à douille de type armoricain comme deux phénomènes contemporains, entretient fâcheusement l'obsolète confusion chronologique.

12 Enfin, la mise en perspective des dépôts armoricains à l'échelle française et plus largement européenne, qui était un des buts majeurs du grand projet de Jacques Briard, fait défaut. On eût apprécié qu'au moins la Normandie voisine, si riche en dépôts de haches à douille de type armoricain dont certains récemment étudiés in situ au cours d'opérations d'archéologie préventive, fût un peu plus prise en compte.

13 Apportant incontestablement une importante documentation graphique inédite tant sur les haches à douille du Bronze final atlantique 3 que sur celles de type armoricain du premier âge du Fer, avec en particulier pour ces dernières des dépôts découverts au $\mathrm{xx}^{\mathrm{e}}$ siècle présentés complets sur le cédérom, l'ouvrage de Josette Rivallain rendra un très utile service aux chercheurs. En revanche, en s'obstinant à défendre contre vents et marées l'idée désormais obsolète d'une production des haches à douille de type armoricain pendant la fin de l'âge du Bronze, il continuera à induire en erreur le grand public comme les étudiants et les chercheurs débutants... 


\section{AUTEURS}

JOSÉ GOMEZ DE SOTO

Directeur de recherche émérite au CNRS, UMR 6566 « CReAAH », Rennes 